\title{
Utilização da telemedicina como estratégia de promoção de saúde em comunidades ribeirinhas da Amazônia: experiência de trabalho interdisciplinar, integrando as diretrizes do SUS
}

\author{
Use of telemedicine technology as a strategy to promote health \\ care of riverside communities in the Amazon: experience \\ with interdisciplinary work, integrating NHS guidelines
}

\author{
Felipe Salles $\mathrm{N}$ eves $\mathrm{M}$ achado ${ }^{1,2}$ \\ M arcela Alves Pinto de Carvalho ${ }^{1,2}$ \\ Andrea M ataresi ${ }^{1,3}$ \\ Eloísa Trevisan M endonça ${ }^{1,4}$ \\ Lucila M oraes Cardoso ${ }^{1,5}$ \\ M ilton Seiyu Yogi ${ }^{6}$ \\ Hamilton M odesto Rigato ${ }^{1}$ \\ M arcelo Salazar ${ }^{1,7}$
}

\footnotetext{
${ }^{1} \mathrm{~N}$ úcleo deApoio à População Ribeirinhada Amazônia(NAPRA). Rua Professor Fernando Thielli 21, Vila Pompéia. 13050472 Campinas SP. felipemac@msn.com ${ }^{2}$ Faculdade de M edicina de Catanduva.

${ }^{3}$ Departamento de Psicologia, Universidade Presbiteriana M ackenzie. ${ }^{4}$ Faculdade de Enfermagem deCatanduva.

${ }^{5}$ Universidade Paulista.

${ }^{6}$ UniversidadeCidade de São Paulo.

${ }^{7}$ Faculdades M etropolitanas Unidas.
}

Abstract This article aims at presenting an experience of professional and medical assistance education using telemedicine as a technology for the promotion and prevention in health care as well as professional qualification of the riverside population living in the margins of $M$ adeira river, in the State of Rondônia. This project comprised a small pole of the telemedicine in thecommunity of Santa Catarina, located by M adeira river, $200 \mathrm{~km}$ from the city of Porto Velho, Rondônia State. Field experience was performed between July 17 and 31, 2006, promoting the inclusion of inhabitants of nine riverside communities. Thesystem allowed si multaneous, real time audio and video exchange providing the riverside communities with unique access to information, medical assistance, and lectures on pre vention in basic health to the population in general. This project showed that the implantation of telemedicine is an alternative for a better distribution of health services. In addition to bringing assistance to people less favoured, it allows social reintegralization of people excluded due to geographic isolation, assists in information diffusion, proves education to the population and future system users, promotes prevention in health, developing responsibility of the population for a better quality of life of the region. Key words Telemedicine, Amazon, Public health, Multisector assistance
Resumo Este artigo apresenta uma experiência de formação educacional e de assistência médica, utilizando a telemedicina como recurso tecnológico para a promoção eprevenção em saúde, além da capacitação profissi onal de populações ribeirinhas do baixo M adeira, no Estado de Rondônia. A atuação contou com a constituição de um pequeno pólo detelemedicina na comunidade deSanta Catarina, localizada no rio M adeira, há duzentos quilômetros do município de Porto Velho. A experiência no campo foi realizada entre 17 e 31 de julho de2006, promovendo a inclusão demoradores de nove comunidades ribeirinhas. 0 sistema permitiu a troca simultânea de vídeo e áudio em tempo real, possibilitando acesso à informação, assi stência médica e palestras sobre prevenção em saúde básica à população em geral. A realização deste projeto mostrou que a implantação da telemedicina é uma alternativa para a melhor distribuição dos serviços de saúde. Além de levar o atendimento a populações menos favorecidas, permite a reintegração social de pessoas preteridas pelo isolamento geográfico, auxilia na difusão de informação, proporciona capacitação aos moradores e futuros usuários do sistema, promovea prevenção em saúde, desenvolvendo a responsabilidade da população para uma melhor da qualidade de vida da região.

Palavras-chave Telemedicina, Amazônia, Saúde coletiva, Atendimento interdisciplinar 
Introdução

O SistemaÚnico deSaúde(SUS) preconizaa universalização do acesso, integralidade da atenção, equidade, descentralização da gestão, hierarquização dos serviços e controle social. A gestão participativa vem somar a esses princípios doutrinários a ampliação dos espaços públicos de construção e pactuação da política de saúde nas esferas governamentais, fortalecendo os espaços instituídos de controle social, mobilizando a população em torno do direito à saúde e, ao mesmo tempo, promovendo a equidade. Esses fatores criam, na estrutura do M inistério da Saúde, um espaço de inclusão e diálogo com grupos populacionais socialmente excluídos ${ }^{1}$. A situação da saúde, no Brasil, passou por inegáveis avanços nos últimos dez anoS, após a implantação do SUS.

Se muitos problemas evoluíram favoravelmente, novos desafios somaram-se aos antigos, exigindo uma estratégia que promova a descentralização, regional ização, tecnologia e informação, no sentido de buscar a construção da integralidade, equidade e atenção humanizada da saúde, que atenda de forma abrangente populações excluídas.

Com a criação e implementação do Programa Saúde da Família (PSF), parte das populações tradicionais da Amazônia, como éo caso de al gumas das populações ribeirinhas, tiveram um acesso à saúde que não possuíam antes. Porém, em se tratando de uma região geográfica de dimensões continentais e sua população se estabelecer ao longo de centenas de rios, na maioria das vezes, sem acesso por estradas, grande parte das pessoas permanecem completamente isoladas. Neste cenário, a telemedicina contribui para associar tecnologia de informação e promoção de saúde, diminuindo a distância dessas populações com os grandes centros.

0 presente estudo aborda a utilização da telemedicina como recurso de diagnóstico edeeducação em saúde nas comunidades ribeirinhas localizadas na região denominada "baixo rio $\mathrm{Ma}$ deira" ao norte do Estado de Rondônia, em uma faixa de 100 à $250 \mathrm{~km}$ de Porto Velho, capital do Estado de Rondônia.

Condições adversas eausência quasetotal dos serviços de saúde de média e alta complexidade, acrescidas do isolamento geográfico e falta de profissionais, acarretam muitas vezes o não cumprimento dos direitos à saúde e a universalidade da assistência.

Este motivo levou o Núcleo de Apoio à População Ribeirinha da Amazônia (NAPRA) ${ }^{2}$ a exe- cutar a primeira experiência completa detelemedicina na Amazônia. O NAPRA é uma associação privada, sem fins lucrativos, que realiza projetos interdisciplinares na Amazônia há mais de dez anos. A missão do NAPRA é dar suporte ao desenvolvimento de comunidades tradicionais em áreas ricas em biodiversidade com ações integradas de saúde, educação e produção e, ao mesmo tempo, contribuir com o desenvolvimento da consciência de trabalho social e interdisciplinar de estudantes universitários integrados ao projeto. As principais atividades do projeto são realizadas todo mês de julho com um grupo de cinquenta a setenta integrantes de diversas universidades e áreas do conhecimento. As principais linhas de atuação são: atendimento médico, odontológico, fisioterapêutico, análises laboratoriais, educação em saúde, formação de educadores ribeirinhos, atividades de ressignificação da cultura local, pesquisas de tecnologias sociais em saúde, educação e produção, suporte à produção e comercialização de produtos florestais não madeireiros (PFNM) e apoio no planejamento das unidades de conservação local. Para cumprir esses objetivos, o NAPRA conta com uma rede de parceiros e colaboradores de diversas empresas e instituições governamentais enão governamentais.

\section{Projeto de telemedicina}

Os objetivos do projeto de telemedicina são:

1. Execução da telemedicina como recurso tecnológico para a promoção e prevenção da saúde em comunidades isoladas, mostrando a viabilidade do atendimento de saúde para populações nessas condições de isolamento;

2. Capacitação profissional deagentes desaúde locais para uso desta tecnologia;

3. Capacitação para o manuseio desta tecnologia aos estudantes universitários durante sua extensão universitária, introduzindo aos estudantes de diferentes instituições e cursos uma realidade diferente daquela vivenciada em sua vida acadêmica;

4. Ser exemplo para a implantação de outros sistemas semelhantes em diferentes regiões do país.

Devido ao pioneirismo e complexidadedesta primeira experiência de telemedicina em comunidades isoladas na floresta amazônica, aliado aos recursos extremamente limitados que o NAPRA dispunha, foi necessária a mobilização de uma rede organizações para a concretização do 
projeto. Os principais atores e suas responsabilidades são:

1. Projeto NAPRA - idealizador, articulador, investidor financeiro eexecutor da experiência;

2. Equipe do Programa de Saúde da Família (PSF) do M unicípio de Porto Velho - disponibilizarão da infra-estrutura das Unidades Básicas de Saúde (UBS);

3. Unidades de serviço hospitalar de atenção terciária - Hospital Escola Emílio Carlos de Catanduva, Hospital Escola Padre Albino de Catanduva - prestação de suporte médico à distância por meio da disponibilizarão de seu corpo clínico;

4. GoDoctor - disponibilização dos softwares, capacitação dos executores e orientação de todo o sistema de telemedicina empregado;

5. Instituto de Telemedicina (ITM S) - equipamentos de eletrocardiograma transtelefônicos, bem como profissionais para pronta análise dos eletros e diagnóstico;

6. Pólo de Telemedicina da Amazônia, Manaus - suporte remoto para as teleconsultas;

7. Telespazio - empresa contratada para estabelecer o link de Internet satelital de alta velocidade e instalação da antena receptora na comunidade;

8. IBAM A-RO e SEM ED (Secretaria M unicipal de Educação de Porto Velho) - suporte logístico;

9. Grupo Jovens com uma missão (JOCU M ) - médico para suporte remoto para as teleconsultas e presencial durante al guns dias;

10. M édicos de diversas instituições do Estado de São Paulo - suporte remoto para as teleconsultas ${ }^{3}$.

\section{Abrangência da experiência}

O Estado de Rondônia conta com 1,31 milhões de habitantes; cerca de um quarto desta população mora na capital. Entre as décadas de sessenta e oitenta do século passado, a população do estado cresceu nove vezes.

O saneamento básico é considerado precário. Segundo o censo de 1998 do IBGE, a rede de esgoto alcançava apenas 3,5\% dos domicílios do estado. Os reflexos dessas condições insalubres aparecem na saúde da população: o estado éconsiderado pela Fundação Nacional de Saúde (FU NASA) uma região endêmica de malária, leishmaniose e febre amarela.

O Sistema Nacional de Vigilância em Saúde (SNVS) publicou, em 2006, um coeficiente geral de mortalidadeinferior a 4,0/1 mil hab., indicando precariedade na cobertura das informações de mortalidade 4 . De acordo com dados do Conselho Federal de M edicina, o estado conta com 4,59 médicos para cada grupo de 10 mil habitantes, menos da metade do queé considerado aceitável pela Organização M undial de Saúde.

Essa situação precária de desenvolvimento se mantém, principalmente, pelo isolamento deseus núcleos populacionais, resultado de difícil acesso e distribuição disforme de seus contingentes 5 .

Devido a essas dificuldades, poucos profissionais se dispõem a trabalhar nestas áreas. Além disso, faltam projetos governamentais efetivos para o desenvolvimento dessas comunidades. $\mathrm{A}$ maioria das iniciativas contemplam questões imediatistas e resultados de curto prazo.

Nesse contexto, as populações ribeirinhas da Amazônia, distantes dos grandes centros, permanecem privadas do acesso a serviços básicos de saúde, educação e a opções que possam auxiliar em seu desenvolvimento, o que leva muitos jovens a emigrarem em busca de melhores condições de vida nos grandes centros populacionais.

Nos 250 quilômetros por via fluvial que separam Porto Velho do distrito de Calama, existem três bases do PSF, localizadas nas comunidades de maior tamanho - São Carlos, Nazaré e Calama. N estas bases, as equipes do PSF atuam de uma a três vezes ao mês nos finais de semana; porém, as UBS das comunidades menores, muitas vezes, ficam meses sem visita, e quando elas acontecem, o médico e o dentista atendem apenas dez consultas pré-agendadas, número insuficiente para a demanda. Em casos de emergência, o paciente precisa ser levado de barco (voadeira com motor 40H P) a Porto Velho, o que depende da disponibilidade de barco, piloteiro e combustível.

Dessa forma, a instalação de um pólo de telemedicina de baixa complexidade (PTBC) no Rio M adeira entre estas bases do PSF, certamente, traria uma melhora significativa no atendimento das necessidades da região, obtendo um modelo de aten ção mais adequado, além de oferecer suporte a uma área de maior abrangência.

\section{D escrição do processo}

O pólo de telemedicina de baixa complexidade (PTBC) do Rio M adeira, instalado na comunidade de Santa Catarina, foi implementado em 2006 naUBS local etrabal hou intensivamenteno período de 17 a 31 dejulho. A escolha desta comunida- 
de, entre as 28 local izadas na região do "baixo rio M adeira", obedeceu os seguintes critérios:

- Representatividade e localização -comunidade típica ribeirinha, cujo acesso se dá unicamente via fluvial a uma distância aproximada de duzentos quilômetros de Porto Velho e equidistante das principais comunidades do chamado baixo rio M adeira;

- Número de famílias - possui 26 famílias, todas cadastradas pelo PSF, número médio de famílias das comunidades da região;

- Estrutura da comunidade - possui gerador elétrico a diesel que funciona 24 horas, telefone público solar, associação de pescadores que permite alojar a equipe, escola em funcionamento e um administrador atuante;

- Recursos na área da saúde - sua UBS, ape sar de ser totalmente de madeira, possui energia el étrica, água tratada e conta com dois agentes de saúde e da visita mensal da equipe de PSF;

- Abrangência do posto de saúde - nove comunidades do entorno dependem do atendimento desta UBS - Santa Catarina, I Iha de Iracema, Tira Fogo, Bomfin, Pombal, São José da Praia, Conceição da Galera, Lago do Caranã e Laranjal -, totalizando aproximadamente mil moradores.

$N$ as proximidades daUBS, foi construída uma base para receber uma antena com conexão de satélite, que trabalhou com a plataforma VSAT IP Banda Larga EVOLV. O sistema permitiu a troca simultânea, em alta velocidade, de vídeo e áudio com qualidade suficiente para o estabelecimento devideoconferências por meio deDSL (Internet banda larga) a Centros deSuporteem Saúde (CSS) dos estados de São Paulo e Amazonas.

Para que toda a consulta e/ ou aula fosse realizada com perfeição de voz e vídeo, foi necessário um ponto de Internet de banda larga conectado em computador com características mínimas ( $1.5 \mathrm{GHz}, 512 \mathrm{M}$ B de memória RAM ), acoplado a uma webcam de alta definição, softwares específicos e um datashow.

Esses centros, em contato com o pólo detelemedicina de baixa complexidade (PTBC) do Rio $M$ adei ra, tinham disponibilidade diária eagenda para consultas com diversos especial istas da saúde. Os serviços de teleducação também funcionaram conforme agenda estipulada.

Com o sistema funcionando, os agentes de saúde informavam as comunidades e seus moradores da possibilidade da consulta médica e faziam triagem dos pacientes. Os estudantes, juntamente com o médico da equipe, examinavamnos e agendavam suas consultas de acordo com a agenda de especialistas gerada, além de operar o sistema. Os agentes do posto de saúde acompanharam a instalação do sistema tecnológico, assim como as consultas, e experimentaram a facilidade do manuseio.

Implementação do sistema de telemedicina - barreiras, desafios epossibilidades

Ante à grande dificuldade de acesso, falta de estrutura, condições climáticas extremas, devido ao calor e chuvas constantes, e ao isolamento geográfico da região, o deslocamento e fixação de profissionais de saúde e de outras áreas de atuação é bastante difícil e custoso. M esmo para o NAPRA, que atua todo mês de jul ho na região, a permanência de médicos com a equipe ao longo de apenas um mês semprefoi um grande desafio. Dessa forma, na busca de uma solução, em junho de 2004, surgiu a idéia de levar para a região formas alternativas de atendimento, aproveitando 0 grande avanço nas tecnologias de informação e detelecomunicações. A partir do contato realizado com a equipe de organização do M obileM ed congresso de mobilidade em saúde, realizado na UNIFESP em São Paulo - , surgiu o primeiro embrião da telemedicina, dando início às conversas que viabilizariam 0 atendimento em saúde via telemedicina já em 2004. A primeira aplicação prática só ocorreu em julho de2005, com a realização deeletrocardiogramas. No entanto, em 2006, conseguiu-se unir um desenho tecnológico adequado aos recursos financeiros captados pelo NAPRA, possibilitando a realização da primeira experiência completa de telemedicina em comunidades ribeirinhas no interior da floresta amazônica. Através dessa experiência, é possível validar e avaliar ações de diferentes profissionais sem os custos, financeiros e pessoais, que implicam 0 deslocamento. A partir desteprojeto, aplicado no rio M adeira, surgiu o conceito do pólo detelemedicina de baixa complexidade (PTBC).

Desde 2004, as barreiras a serem vencidas foram inúmeras, começando pelo desenho tecnológico do sistema e identificação de empresas de telecomunicações dispostas a enfrentar o desafio. A princípio, o sistema todo tinha um alto custo e uma logística muito complicada, inviabilizando sua operacionalização, na medida em que estavam previstas instalações de três antenas comunicando-se entresi para que o sistema funcionasse. No entanto, uma nova solução (mais barata e prática) foi desenhada, o que viabilizou o projeto. Outra barreira era convencer os diversos parcei- 
ros queuma ONG, relativamente pequena ecomposta em sua maioria por estudantes universitários e recém-formados, tinha know-how e persistência para realizar este projeto. Com o comprometimento de alguns dos parceiros e parte dos recursos, o passo seguinte foi a elaboração de um plano de ação detalhado, definição da logística e negociação detodos os contratos de serviços para viabilizar a experiência. Nesta fase, foram utilizadas as tecnologias móveis e de videoconferência, utilizando os recursos de I nternet disponíveis nas cidades - a empresa que forneceu o link e antena estava no Rio de Janeiro (RJ), a antena, em Curitiba (PR), os representantes da empresa GoD octor em M anaus (AM) e São Paulo (SP) e os integrantes do NAPRA espalhados em cidades do Estado de São Paulo e em Londres, Inglaterra. Também ajudaram, na especificação do sistema, parceiros de Porto Velho (RO) e representantes das comunidades ribeirinhas diretamente do rio $M$ adeira. Não houvenenhuma reunião presencial com envolvidos para a implantação e funcionamento do sistema.

Em campo, na comunidade de Santa Catarina no rio $M$ adeira, os desafios apresentaram uma dinâmica de solução completamente diferente. Depois de definido o local para a instalação dos equipamentos, os primeiros desafios de campo foram o transporte da antena para a comunidade de Santa Catarina no rio M adeira e sua instalação e estabelecimento do link com o satélite da Telespazio. Tarefas consideradas simples na cidade, como a construção de uma base de concreto para uma antena, telefonar para o Rio de Janeiro ou comprar um cabo de Internet demoram dias ou até semanas. Transpostos os obstáculos de instal ação econectividade, com apenas cinco dias de atraso no nosso planejamento, estávamos conectadosà Internet no meio da floresta Amazônica, há duzentos fluviais da cidade mais próxima. Depois de al guns testes e ajustes nos softwares, foi realizada a primeira consulta, um momento histórico e de grande emoção para os integrantes do NAPRA, médicos de suporte remoto e, sobretudo, para o primeiro paciente- um ribeirinho que nunca havia sido consultado por um médico.

Com o sistema pronto e testado, efetuou-se o refinamento da logística para avisar as comunidades vizinhas, a definição da grade de atendimentos com os diver sos especial istas de São Paulo e $M$ anaus e a operacionalização do planejamento previamente realizado para os atendimentose aulas.

O pólo detelemedicina de baixa complexidade (PTBC) tornou-se, então, uma realidade e, desde os primeiros momentos, observou-se que é um sistema expansível, podendo acoplar-se a muitos recursos disponíveis natelemedicina, entreoutros, telexames, teleprontuários, teledermatologia, telepsiquiatria. 0 sistema também possibilita a formação de agentes comunitários, líderes da comunidade e demais profissionais de saúde por meio de software e programas de teleducação e educação continuada ${ }^{6}$.

0 custo dos equipamentos para um mês de atendimento ficou em aproximadamente $\mathrm{R} \$$ 30.000,00 por ponto; 0 custo do link satelital pode diminuir consideravelmente com a contratação de diversos pontos ou por um período contínuo.

\section{Resultados}

A telemedicina é uma das opções para prestar assistência médica a pacientes que estão geograficamentedistantes do médico. Além disso, éuma forma de difundir cuidados na área da Saúde para localidades desprovidas destes serviços ou, ainda, deficitários de determinados tipos de procedimentos. 0 objetivo é permitir igualdade de acesso aos serviços médicos, independente da localização geográfica do indivíduo.

A telemedicina é um serviço emergente que apresentou contribuições efetivas na prestação de cuidados de saúde, facilitando o acesso a cuidados médicos de melhor qualidade, obtenção de uma segunda opinião médica a custos acessíveis, proporcionando extensão de serviços médicos especializados a locais remotos.

Através de uma estrutura permanente, vislumbra-se a criação de um protocolo de atendimento com horários e dias fixos para cada especialidade, possibilitando que as comunidades próximas marquem consultas por meio de seus agentes comunitários. Um dos resultados diretos desta proposta é a diminuição dos deslocamentos dos pacientes até Porto Vel ho para serem atendidos, contribuindo para a descentralização e diminuindo a demanda de atendimento nos hospitais públicos da capital.

Outro ben efício éa troca de experiências entre universitários eprofissionais de diferentes regiões do Brasil, que poderiam utilizar esterecurso para aumentar suas atividades acadêmicas, além deser um incentivo para a maior participação no desenvolvimento do seu próprio estado, buscando alternativas para outros problemas locais.

Os resultados e benefícios diretos podem ser facilmente medidos e apresentados; os indiretos são difíceis de mensurar, pois envolvem a incor- 
poração da tecnologia para os acadêmicos e para a população. Com relação ao SUS, observamos que foram promovidos e experimentados seus princípios doutrinários eorganizacionais, foram incluídos os participantes e beneficiados no projeto, levantados os indicadores de saúde e as doenças de notificação compulsória. Como promotores de extensão acadêmica, os integrantes foram expostos a diferentes culturas e à divergência regional das doenças num país composto por diversos cenários, capacitando-os para uso desta nova tecnologia.

Resultados da atuação da telemedicina na comunidade de Santa Catarina no rio Madeira (RO)

Das oitenta consultas realizadas na UBS de Santa Catarina ("Amazônia"), 34 foram teleconsultas e/ou segunda opinião médica. Participaram treze profissionais à distância ecinco profissionais locais, totalizando onze especialidades. A equipe de saúde do NAPRA contou com 44 profissionais - oito formadose 36 estudantes denove universidades diferentes, totalizando dezessete cursos.

Foram realizadas, ainda, cinco aulas a distância para públicos diversos:

. Duas sobre anti-inflamatórios com o Prof. Dr. Marcelo Mascará do ICB 1-USP para integrantes do NAPRA;

. Uma sobre malária com o Dr. Franklin Simões do Centro de Telemedicina de M anaus;

- Duas para estudantes da comunidade sobre higiene básica com o Prof. Dr. Milton Yogi de São Paulo.

Potencial inovador com marcas e diretrizes do participativo do SUS

A utilização deum sistema tecnológico na promoção da saúde em regiões de difícil acesso se mostra promissora. Desenvolver tal sistema implica capacitar e integrar membros das comunidades para que continuem o trabalho, deixando de ser somente público-alvo. Além desta capacitação, a utilização dos recursos de áudio e vídeo possibilita a realização de aulas e pal estras para a população, promovendo qualidade de vida, tornando-a protagonista de bons resultados.

Um país de proporções continentais como o Brasil pode usufruir da tecnologia para comunicar-se de um extremo a outro. A atribuição da tecnologia em saúde garante maior abrangência e acesso, em lugares mais distantes, à saúde, edu- cação e preven ção ${ }^{7}$, fazen do com que sejam cumpridos os direitos da população de ser atendida.

\section{Caráter multiplicador}

Esta experiência pode ser replicada para diversas outras comunidades ribeirinhas isoladas no interior da floresta Amazônica e em outros biomas brasileiros, desde que haja um esforço inicial de implantação da antena e dos equipamentos de informática, bem como no aprimoramento dos métodos de treinamento de multiplicadores locais e desenvolvimento demais parceiros para 0 atendimento remoto.

\section{Caráter interdisciplinar}

A instalação desse sistema para telemedicina abre caminhos para uma série de outras melhorias nas comunidades ribeirinhas, como a ampliação do suporte a educadores locais - formando-os de forma constante e dialogando sobre as dificuldades cotidianas no processo de ensinoaprendizagem (teleducação) -, e a realização de reuniões para negociações de produtos florestais não madeireiros locais, inserindo esses produtos em outros mercados nacionais e internacionais. Além disso, a implantação de um sistema que funciona para a telemedicina pode funcionar também para a comunicação de crimes ambientais e monitoramento de indicadores de preservação da biodiversidade. Finalmente, a implementação desta ferramenta de comunicação, além de melhorar 0 atendimento à saúde, possibilitar diversas melhorias para as comunidades.

\section{Discussão}

No Estado de Rondônia, existe uma grande dificuldade na assistência à saúde nas comunidades situadas em áreas geograficamente distantes. Esta assistência, muitas vezes, é baseada em centros urbanos, cuja demanda por atendimento especializado não justifica o custo da manutenção de profissionais e equipamentos específicos para a atenção médica terciária.

No geral, essas comunidades contam somente com a assistência primária de saúde, oferecida pela rede pública através de unidades dotadas de profissionais generalistas em medicina, odontologia e enfermagem, além de agentes comunitários do PSF.

Com o avanço das tecnologias de informação e telecomunicações, a possibilidade da inserção 
da telemedicina, como um dos instrumentos de acesso a populações isoladas, está cada vez mais próxima da realidade, possibilitando, além de outros benefícios, a expansão da assistência terciária em saúde. É imprescindível a continuidade do projeto, bem como a ampliação da sua praticidade, beneficiando ainda mais as comunidades ribeirinhas, por meio de novas políticas públicas que facilitem sua implantação e manutenção.

\section{Conclusão}

A aplicação da telemedicina na Amazônia encurta a distância geográfica no queser efereao desenvolvimento sustentado de suas comunidades, melhorando a qualidade do atendimento e a efetividade de sistemas de saúde. Além disso, permite:

. o contato direto e simultâneo com centros avançados em saúde;

- a melhoria da orientação de suporte à vida frente a situações de risco;
- a melhoria da resolubilidadedo atendimento primário e secundário;

- o acompanhamento de especialista, uma vez que 0 atendimento secundário é realizado apenas na zona urbana (Porto Velho-RO);

- a prevenção em saúde, utilizando a teleducação e educação continuada favorecida pelos recursos audiovisuais.

Dessemodo, a implantação da telemedicina é uma alternativa para a melhor distribuição dos serviços de saúde, por que leva atendimento a quem necessita, reintegra à sociedade pessoas preteridas pelo isolamento geográfico, auxilia na difusão de informação, proporciona capacitação de moradores e futuros usuários do sistema, promovea prevenção em saúdee desenvolve, com isso, a responsabilidade da população para uma melhora da qualidade de vida da região.

A telemedicina é uma ferramenta necessária para a complementar o sistema de saúde de regiões isoladas, uma vez que amplia a informação e o conhecimento, melhorando a saúde do individuo e da comunidade.

\section{Colaboradores}

FSN M achado trabalhou na coordenação, atuação local na Amazônia, pesquisa e redação do texto. M AP Carvalho, ET M endonça e A M ataresi colaboraram com a pesquisa, atuação local na Amazônia e redação do texto. LC M oraes trabaIhou na pesquisa, metodologia e redação do texto. M S Yogi colaborou com o apoio técnico, desenho do sistema, orientação tecnológica e de pesquisa, atividades do sistema à distância (São Paulo). M Salazar idealizou o sistema e coordenou o projeto, participou da atuação local na Amazônia, sendo corresponsável pela logística, contratações e desenvolvimento das parcerias, redação de trechos e revisão final do presente artigo eH M Rigato foi um dos idealizadores do sistema e coordenador do projeto, participando da atuação local na Amazônia, sendo corresponsável pela logística, contratações e desenvolvimento das parcerias. Revisou o presente artigo. Todos os autores participaram ativamente das discussões para o planejamento, operação e resultados desta experiência. 
Agradecimentos

Em primeiro lugar, agradecemos à população da comunidade de Santa Catarina do baixo rio madeira (Porto Velho-RO), que confiou no projeto e nos recebeu com carinho, não poupando esforços para ajudar na implementação do sistema.

A experiência com utilização da telemedicina para o diagnóstico de casos clínicos e de educação em saúde contou com apoio financeiro da Fundação Padre Albino (UNIFPA), da participação dealunos da Faculdade de $M$ edicina de $C$ atanduva (FAM ECA) e Faculdade de Enfermagem de Catanduva (FEC), da disponibilização do corpo clínico dos hospitais Emilio Carlose H ospital Escola Padre Albino. Além disso, contribuíram muito com sua experiência alguns médicos da Universidade Estadual do Amazonas, representantes do Consel ho Federal deM edicina, integrantes do Pólo de Telemedicina da Amazônia baseado em M anaus. A empresalTM Sforneceu os equipamentos para a realização de eletrocardiograma de forma remota; a secretaria de saúde de Porto Velho autorizou a utilização dos postos de saúde locais; o IBAM A eSecretaria de Educação dePorto Velho deram apoio logístico. Agradecemos, ainda, a Telespazio, empresa contratada para o estabelecimento do link entre São Paulo, M anaus ea comunidade de Santa Catarina; a empresa GoDoctor, que prestou assistência e consultoria no dimensionamento da tecnologia, instalação e testes desoftware; além de uma rede de profissionais colaboradores que prestaram suporte remoto. Finalmente, agradecemos aos voluntários, profissionais e apoiadores que tornaram possível a atuação do NAPRA no ano de 2006.

\section{Referências}

1. Brasil. Ministério da Saúde. Secretaria de Gestão Estratégia e Participativa. Departamento de Apoio à Gestão Participativa. [acessado 2006 set 14]. Disponível em: http://portal.saude.gov.br/portal/sgp/ visualizar_texto.cfm?idtxt $=22736$

2. Mariotti $\bar{A} C H$. Projeto NAPRA: núcleo de apoio à população ribeirinha da Amazônia X etapa. 2004. [acessado 2006 mar 17]. Disponível em: http:// www.napra.org.br

3. Núcleo de Apoio à População Ribeirinha da Amazônia. [acessado 2006 set 18]. Disponível em: http:/ /www.napra.org.br

4. Brasil. M inistério da Saúde. Secretaria de Vigilância em Saúde. Sistema nacional de vigilância em saúde: relatório de situação: Rondônia/M inistério da Saúde, Secretaria de Vigilância em Saúde. 2ª ed. Brasília: M inistério da Saúde; 2006.

5. Secretaria do M eio Ambiente do Estado de Rondônia. Companhia de Tecnologia de Saneamento Básico. Considerações sobre o zoneamento socioeconômico-ecológico do Estado de Rondônia. Porto Velho. 2004 [acessado 2006 mar 17]. Disponível em: http:/ /www.rondonia.ro.gov.br/revistas/zoneamento

6. Seabra ALR, Pitta GBB, Gomes MAM. Telemedicina: uma proposta para assistência e educação continuada no Pólo Saúde da Família em Alagoas. [acessado 2006 set 18], Disponível em: http://www. ministerio.saude.bvs.br

7. Chao LW, Silveira PSP, Bohn GM. Discipline of telemedicine, Faculty of M edicine of the University of São Paulo, Brazil. [acessado 2006 set 14]. Disponível em: http://www.dim.fm.usp.br/telemed/postelem.php

Artigo apresentado em 23/12/2006

Aprovado em 18/06/2007

Versão final apresentada em 19/06/2007 